\title{
DISTANT RADIO GALAXIES: PROBES OF THE FORMATION OF MASSIVE GALAXIES
}

\author{
HUUB RÖTTGERING, PHILIP BEST, LAURA PENTERICCI AND \\ GEORGE MILEY \\ Leiden Observatory, \\ P.O. Box 9513, 2300 RA Leiden, The Netherlands
}

\begin{abstract}
In this paper we review some of the evidence that the hosts of powerful high redshift $(1 \lesssim z \lesssim 5$ ) radio galaxies (HzRGs) are the progenitors of present day brightest cluster galaxies (BCGs). On the basis of HST imaging we argue that the scenario describing the formation of BCGs consists of at least two important stages. At $z>2$ a significant fraction of the stellar mass of a BCG is formed during a massive burst of star-formation. By $z \sim 1$, well developed massive ellipticals are already observed and from then until the present epoch, the total mass in stars of the BCG will further grow by a factor of order 3, mainly through the accretion of cluster ellipticals.
\end{abstract}

\section{Introduction}

During the last decade, evidence has been accumulated that powerful radio galaxies at large distances $(1 \lesssim z \lesssim 5)$ are hosted in galaxies that are the most massive for their epoch and are situated in a dense, cluster type environment (Best et al. 1997a). There are now more than 140 radio galaxies with $2<z<4.5$ known (e.g. Röttgering et al. 1997). This sample of $z>2$ radio galaxies combined with the large number of known $z \sim 1$ radio galaxies therefore forms an ideal tool to study the evolution of brightest cluster galaxies from $z=5$ down to the present epoch.

In this paper we will first review briefly some of the basic properties of distant radio galaxies, with particular emphasis on the question of whether or not these high redshift radio sources are located in cluster-like environments. Subsequently, two $H S T$ imaging projects are discussed, one focusing on $z \sim 1$ radio galaxies and the other on $z>2$ radio galaxies. Finally, we 
will show that the observations do indeed sketch a picture of how the assembly of massive galaxies from $z=5$ to the present epoch has taken place.

\section{Properties of $z>2$ radio galaxies}

Since in this paper we would like to discuss the use of distant radio galaxies as probes of the formation of massive galaxies, two properties of $z>2$ HzRGs are of particular relevance: their total mass in stars, and the rate at which they form stars.

There are two fundamental difficulties in obtaining a robust estimate for the number of stars in these systems. Firstly, the best wavelength region to probe the bulk of the stellar population is around $1-2 \mu \mathrm{m}$ rest-frame. For the HzRGs this region is red-shifted towards the thermal infrared, a region in which it is difficult to carry out observations with sufficient sensitivity. Secondly, the radio galaxies have an emission component, with a blue spectrum, which is aligned with the radio source. This blue light is likely to be due to a combination of young stars, scattered light from a hidden quasar and nebular continuum emission from the emission line gas. Despite these difficulties, the spectrum of HzRGs indicates that they contain a stellar mass which is generally within a factor of a few of $5 \times 10^{11} M_{\odot}$ (McCarty et al. 1992; Eales and Rawlings 1996).

In several HzRGs the UV continuum is probably dominated by young stars, and the estimated star-formation (SF) rates can then be directly estimated (Madau et al. 1996). SF rates of up to a $1000 M_{\odot} \mathrm{yr}^{-1}$ are found (Dey et al. 1997). In three distant radio galaxies submillimeter continuum emission has been detected (Hughes et al. 1997). Since this dust is likely to be heated by young stars, this submillimeter detection directly implies $\mathrm{SF}$ rates of well over $1000 M_{\odot} \mathrm{yr}^{-1}$. These two estimates give similar high rates for the SF indicating that distant radio galaxies can indeed form stars at such a high rate. It further indicates that dust and young stars are distributed in such a way that despite the copious amount of dust a large fraction of the UV light emitted by the young stars can nevertheless escape the galaxy.

It is now well established that at least some HzRGs at $z \sim 1$ are located in fairly massive clusters (Dickinson 1997). At higher redshifts the evidence is more tentative, but clearly building up; in a number of cases companion galaxies to the HzRGs are detected (for a review see Dickinson 1997). Of order $20 \%$ of the radio galaxies have large rotation measures (RM), strongly suggesting that the radio sources are surrounded by hot magnetized cluster gas (Carilli et al. 1997). And indeed, the HzRGs with the highest RM, 1138-262 at $z=2.156$ (Pentericci et al. 1997), was recently detected with 
ROSAT, indicating an amount of hot gas present similar to that of the cluster around Cygnus A (Carilli et al. 1998).

\section{3. $H S T$ imaging of $28 z \sim 1$ 3CR radio Galaxies}

During the last 4 years we have carried out a detailed study of a virtually complete sample of $28 z \sim 13 \mathrm{CR}$ radio galaxies using the $H S T$, UKIRT and the VLA (Best et al. 1997a;1997b). From a detailed analysis, we concluded that the magnitudes, colours and profiles are all consistent with the host of the radio galaxy being a massive $\left(M_{\star} \sim 5 \times 10^{11} M_{\odot}\right)$ elliptical galaxy that formed at high redshift $(z>3-5)$. For a number of reasons, we suggest that a significant fraction of the excess blue light is due to young stars, indicating that that these systems must be forming stars with a rate of order $f \times 50-100 M_{\odot} \mathrm{yr}^{-1}$, where $f$ is the fraction of the excess blue light that is due to stars. If this star burst last for $10^{7}$ years, then the total mass in the stars being formed is $\sim f \times 10^{9} M_{\odot}$, a small fraction of the mass in stars already present.

\section{HST imaging of $z>2$ radio galaxies}

To study the formation of massive galaxies using radio galaxies as probes we are observing the continuum emission of 10 of the most distant radio galaxies with WFPC2 on the HST. Our first impression is that there is a large diversity in the morphologies of the objects. The degree of clumpiness and the overall alignments with the axis of the radio source vary greatly from object to object. An extreme case is that of 1138-262 that contains of order 10 clumps. These clumps have typical sizes in the range of $2-10 \mathrm{kpc}$, have profiles that can not be well fit by either an $\mathrm{r}^{1 / 4}$ or an exponential law and have star formations rates of order $1-5 M_{\odot} \mathrm{yr}^{-1}$. Interestingly, all these characteristics are similar to those of the UV-dropout galaxies at $z \sim 3$ (Giavalisco et al. 1996). A first comparison with the HST images of the $z \sim 13 \mathrm{CR}$ radio galaxies, indicates that in general the most distant radio galaxies contain a factor of $2-3$ more of these star forming clumps.

\section{The assembly of massive galaxies}

The observations discussed suggest a scenario for the formation of BCGs that contains at least two important stages. At $z>2$, the high starformation rates as inferred from the UV-continuum and dust emission measurements, suggest the presence of a starburst that is so vigorous that we are indeed witnessing the formation of major parts of the galaxies. The stars in the galaxy then settle in the potential well and at $z \sim 1$ we can observe the fully developed ellipticals hosting the powerful $3 \mathrm{CR}$ radio sources. 
Interestingly, the brightest cluster galaxies at $z \sim 0$ are about one magnitude more luminous at K-band than the $z \sim 1$ galaxies. If the hosts of distant $3 \mathrm{CR}$ galaxies are indeed the precursors of present day BCGs, then the 3CR galaxies must grow by about a factor 3 in stellar mass between $z \sim 0$ and $z \sim 1$. This can not be done by actively forming a significant amount of new stars during this period, basically because the $z \sim 0$ would be much bluer than observed. The most natural way of doing this is by accreting faint cluster ellipticals onto the 3CR galaxy. From the observations of a sample of BCG associated with known rich clusters up to $z \sim 1$, Aragón-Salamanca et al. (1997) also suggest that vigorous accretion is a dominant process during the formation of BCGs. They further show that such a scenario can be understood quantitatively using semi-analytical modeling techniques.

The naive interpretation from the observations therefore indicate that a vigorous starburst and the subsequent accretion of dwarf ellipticals are both important processes in the formation of BCGs. However, in a number of $z \sim 1$ radio galaxies and in the case of 1138-262 X-ray emission has been observed, presumably from hot cluster gas. If in this gas a cooling flow has developed, then the amount of gas cooling must be enormous and will have an impact on the evolution of the BCGs.

Concluding, if all these three mechanisms contribute in the formation of BCGs, then there is no "epoch of formation" of BCGs, but the formation of BCGs is an ongoing process during the history of the universe.

\section{References}

Aragón-Salamanca A., Baugh C., Kauffmann G., 1997, in S. D’Odorico, A. Fontana and E. Giallongo (eds.), The Young Universe, A.S.P. Conf. Ser, in press, astro-ph $/ 9711146$ Best P., Longair M. S., Röttgering H. J. A., 1997a, MN, in press, astro-ph/9709195

Best P., Longair M. S., Röttgering H. J. A., 1997b, MN, in press, astro-ph/9707337

Carilli C. L., Harris D. E., Pentericci L., Röttgering H., Miley G. K., Bremer M. N., 1998, ApJL: submitted

Carilli C. L., Röttgering H., van Ojik R., Miley G. K., van Breugel W., 1997, ApJS, 109,1

Dey A., Van Breugel W., Vacca W. D., Antonucci R., 1997, ApJ, 490, 698

Dickinson M., 1997, in L. Da Costa (eds.), the ESO/VLT meeting: Galaxy Scaling Relations: Origins, Evolution and Applications, in press, astro-ph/9612178

Eales S. A., Rawlings S., 1996, ApJ, 460, 68

Giavalisco M., Steidel C. C., Macchetto F. D., 1997, ApJ, 470, 189

Hughes D. H., Dunlop J. S., Rawlings S., 1997, MNRAS, 289, 766

Madau P., Ferguson H. C., Dickinson M. E., Giavalisco M., Steidel C. C., Fruchter A., 1996, MNRAS, 283, 1388

McCarthy P. J., Persson S. E., West S. C., 1992, ApJ, 386, 52

Pentericci L., Röttgering H., Miley G. K., Carilli C. L., McCarthy P., 1997, A\&A, 326, 580

Röttgering H., van Ojik R., Miley G., Chambers K., van Breugel W., de Koff S., 1997, A\&A, 326, 505 